\title{
On using sinc collocation approach for solving a parabolic PDE with nonlocal boundary conditions
}

\author{
Mohamed El-Gamel*, Mahmoud Abd El-Hady \\ Department of Mathematical Sciences, Faculty of Engineering, Mansoura University, Egypt.
}

\begin{abstract}
This work suggests a simple method based on a sinc approximation at sinc nodes for solving parabolic partial differential equations with nonlocal boundary conditions. Sinc approximation are typified by errors of the form $\mathrm{O}\left(e^{-k / h}\right)$, where $k>0$ is a constant and $h$ is a step size. Some numerical examples are utilized to reveal the efficaciousness and precision of this method. The suggested method is flexible, easy to programme and efficient.
\end{abstract}

Keywords: Sinc function, nonlocal, collocation, numerical solutions.

2020 MSC: 65L60, 45J05.

(C)2021 All rights reserved.

\section{Introduction}

In the last decades, the development of numerical techniques for the solution of the nonlocal boundary value problems has been an important research topic in many branches of science and engineering. Particularly in viscoelasticity, it has been the subject of some recent works [10-12]. The nonlocal problems are very important in the transport of reactive and passive contaminates in aquifer, an area of active interdisciplinary research of mathematicians, engineers, and life scientists. We refer the reader to [13, 14] for the derivation of mathematical models and for the precise hypothesis and analysis.

Mathematical formulation of this kind also arises naturally in various engineering models, such as nonlocal reactive transport in underground water flows in porous media [13, 15], heat conduction, radioactive nuclear decay in fluid flows [34], non-Newtonian fluid flows, semiconductor modelling [2], and biotechnology. Also, the number of physical phenomena modeled by partial differential equations (PDEs) involving nonlocal integral terms is constantly increasing. Certain problems arising in thermodynamics, in the quasistatic theory of thermoelasticity [16, 17, 26], heat conduction [5-7, 9, 29], and plasma physics [35], can be reduced to the nonlocal problems with integral condition. The above mentioned papers consider problems with parabolic equations. However, some problems concerning the dynamic of ground waters are described in terms of hyperbolic equations [31, 42].

\footnotetext{
${ }^{*}$ Corresponding author

Email addresses: gamel_eg@yahoo.com (Mohamed El-Gamel), mahmoud_ibrahim87@yahoo.com (Mahmoud Abd El-Hady)

doi: $10.22436 /$ jnsa.014.01.04
}

Received: 2018-11-28 Revised: 2020-03-29 Accepted: 2020-04-08 
The numerical treatment for PDEs with different kinds of nonlocal conditions has a great interest such as Finite difference algorithms [18], finite element approximations [25, 33], spectral collocation method [3], Galerkin techniques [8], collocation approaches [27], Tau schemes [44] and sinc-collocation method [45]. Existence, uniqueness and some properties of the solution to these problems were established in $[16,17,28]$.

Sinc methods are highly efficient numerical methods developed by Stenger [37, 39], El-Gamel [19, 20, $23,24]$ and others $[4,32,36,43]$.

Using collocation method based on sinc function we introduce the solution to parabolic equations given by

$$
u_{t}=u_{x x}+\mu_{1}(x) u_{x}+B(x) u+q(x, t), \quad 0<x<1, \quad 0<t \leqslant 1,
$$

subject to the initial condition

$$
u(x, 0)=f(x), \quad 0<x<1,
$$

and subject to nonlocal boundary conditions

$$
\begin{gathered}
a_{0}(t) u(0, t)+a_{1}(t) u_{x}(0, t)+a_{2}(t) u(1, t)+a_{3}(t) u_{x}(1, t) \\
+\sum_{j=0}^{p} \alpha_{j}(t) u\left(\epsilon_{j}, t\right)+\int_{0}^{1} k_{1}(x, t) u(x, t) d x=g_{1}(t), \\
\left.b_{0}(t) u(0, t)+b_{1}(t) u_{x}(0, t)+b_{2}(t) u(1, t)+b_{3}(t) u_{x}(1, t)\right] \\
+\sum_{j=0}^{p} \beta_{j}(t) u\left(\xi_{j}, t\right)+\int_{0}^{1} k_{2}(x, t) u(x, t) d x=g_{2}(t),
\end{gathered}
$$

where $x$ and $t$ are the spatial and time coordinates, respectively, $u(x, t)$ is unknown function to be determined, $q, f, k_{1}, k_{2}, g_{1}, g_{2}, a_{i}, b_{i}, i=0,1,2,3, \alpha_{i}, \beta_{i}, i=0,1, \ldots, p$ are known functions and $\epsilon_{i}$, $\xi_{i} \in(0,1), i=0,1, \ldots, p$.

This paper has the following organization. In Section 2, an excellent summary of sinc methods and their proofs is briefly referenced. In Section 3, the sinc collocation method is introduced and developed to solve parabolic partial differential equations with nonlocal conditions. Some numerical results are presented in Section 4 to show the efficiency of the proposed method. Finally, a conclusion of the study is presented in Section 5 .

\section{Preliminaries}

As was already mentioned in the above introduction, a general review of sinc function approximation is given in $[30,38]$ and the recent papers (see $[1,21,22]$ for excellent surveys).

\section{Description of the sinc-collocation scheme}

A first step is to discretize the time variable at $t=t_{i}:=i \Delta t$, where $\Delta t$ is a time step and $i=0,1,2, \ldots$. Letting $u_{i}(x):=u\left(x, t_{i}\right)$, then we have the following finite difference approximation to $\partial_{t} u$ :

$$
\frac{\partial u}{\partial t} \approx \frac{u_{i}-u_{i-1}}{\Delta t}
$$

By substituting Eq. (3.1) into Eq. (1.1), we obtain the following linear ordinary differential equation at $\mathrm{t}=\mathrm{t}_{\mathrm{i}}$

$$
\mathbf{L} u_{i}=\sum_{j=0}^{2} \mu_{j}(x) \frac{d^{j} u_{i}}{d x^{j}}=M\left(x, t_{i}\right),
$$


where

$$
\mu_{2}(x)=1, \quad \mu_{0}(x)=B(x)-\frac{1}{\Delta t}, \quad \text { and } \quad M\left(x, t_{i}\right)=-q\left(x, t_{i}\right)-\frac{u_{i-1}}{\Delta t} .
$$

Also, hence, the initial condition from equation (1.2), we have

$$
u_{0}=u(x, 0)=f(x)
$$

and the boundary condition from equation (1.3) and (1.4), we have

$$
\begin{array}{r}
a_{0}\left(t_{i}\right) u_{i}(0)+a_{1}\left(t_{i}\right) u_{i}^{\prime}(0)+a_{2}\left(t_{i}\right) u_{i}(1)+a_{3}\left(t_{i}\right) u_{i}^{\prime}(1) \\
+\sum_{j=0}^{p} \alpha_{j}\left(t_{i}\right) u_{i}\left(\epsilon_{j}\right)+\int_{0}^{1} k_{1}\left(x, t_{i}\right) u_{i} d x=g_{1}\left(t_{i}\right), \\
b_{0}\left(t_{i}\right) u_{i}(0)+b_{1}\left(t_{i}\right) u_{i}^{\prime}(0)+b_{2}\left(t_{i}\right) u_{i}(1)+b_{3}\left(t_{i}\right) u_{i}^{\prime}(1) \\
+\sum_{j=0}^{p} \beta_{j}\left(t_{i}\right) u_{i}\left(\xi_{j}\right)+\int_{0}^{1} k_{2}\left(x, t_{i}\right) u_{i} d x=g_{2}\left(t_{i}\right) .
\end{array}
$$

Let the solution $u\left(x, t_{i}\right)$ of the problem (3.2) be approximated by

$$
u_{i, A}(x)=c_{-N-2}^{i} \Phi_{1}(x)+c_{-N-1}^{i} \Phi_{3}(x)+u_{m}^{i}(x)+c_{N+1}^{i} \Phi_{4}(x)+c_{N+2}^{i} \Phi_{2}(x), \quad A=2 N+5,
$$

where

$$
u_{i, m}(x)=\sum_{j=-N}^{N} \frac{s_{j}(x)}{\phi^{\prime}(x)} c_{j}^{i}
$$

where $S_{j}(x)$ is the function $S(j, h) \circ \phi(x)$ for some fixed step size $h$ and the boundary basis functions $\Phi_{1}, \Phi_{2}, \Phi_{3}$ and $\Phi_{4}$ are cubic Hermite functions given by

$$
\Phi_{1}(x)=(2 x+1)(1-x)^{2}, \quad \Phi_{2}(x)=(-2 x+3) x^{2}, \quad \Phi_{3}(x)=x(1-x)^{2}, \quad \Phi_{4}(x)=-x^{2}(1-x),
$$

via the identities

$$
\begin{array}{llll}
\Phi_{1}(0)=1, & \Phi_{2}(0)=0, & \Phi_{3}(0)=0, & \Phi_{4}(0)=0, \\
\Phi_{1}^{\prime}(0)=0, & \Phi_{2}^{\prime}(0)=0, & \Phi_{3}^{\prime}(0)=1, & \Phi_{4}^{\prime}(0)=0, \\
\Phi_{1}(1)=0, & \Phi_{2}(1)=1, & \Phi_{3}(1)=0, & \Phi_{4}(1)=0, \\
\Phi_{1}^{\prime}(1)=0, & \Phi_{2}^{\prime}(1)=0, & \Phi_{3}^{\prime}(1)=0, & \Phi_{4}^{\prime}(1)=1,
\end{array}
$$

and

$$
u_{i, m}(0)=u_{i, m}(1)=0, \quad \text { where } \quad \phi^{\prime}(x)=\frac{1}{x(1-x)} .
$$

Substituting (3.5) into (3.2), we get

$$
\mathbf{L} u_{i, m}(x)=\sum_{j=0}^{2} \mu_{j}(x) \frac{d^{j} u_{i, m}}{d x^{j}}=\widetilde{M}\left(x, t_{i}\right),
$$

where

$$
\widetilde{M}\left(x, t_{i}\right)=M\left(x, t_{i}\right)-c_{-N-2}^{i} L \Phi_{1}(x)-c_{-N-1}^{i} L \Phi_{3}(x)-c_{N+1}^{i} L \Phi_{4}(x)-c_{N+2}^{i} L \Phi_{2}(x),
$$

and

$$
\begin{aligned}
& u_{i, m}^{\prime}(x)=\sum_{j=-N}^{N}\left[-\frac{\phi^{\prime \prime}(x) S_{j}(x)}{\left[\phi^{\prime}(x)\right]^{2}}+\frac{S_{j}^{(1)}(x)}{h}\right] c_{j}^{i}, \\
& u_{i, m}^{\prime \prime}(x)=\sum_{j=-N}^{N}\left[\frac{\left(\phi^{\prime \prime}(x)\right)^{2}-\phi^{\prime \prime \prime}(x) \phi^{\prime}(x)}{\left[\phi^{\prime}(x)\right]^{3}} S_{j}(x)-\frac{\phi^{\prime \prime}(x)}{\phi^{\prime}(x)} \frac{S_{j}^{(1)}(x)}{h}+\frac{\phi^{\prime}(x)}{h^{2}} S_{j}^{(2)}(x)\right] c_{j}^{i} .
\end{aligned}
$$


Theorem 3.1. If the assumed approximate solution of parabolic PDE with nonlocal boundary conditions (1.1) is (3.5), then the discrete sinc-collocation system is given by

$$
\sum_{j=-N}^{N}\left[\sum_{p=0}^{2} g_{p}\left(x_{k}\right) \frac{\delta_{j k}^{(p)}}{h^{p}}\right] c_{j}^{i}=\widetilde{M}\left(x_{k}, t_{i}\right), \quad k=-N-1,-N, \ldots, N+1,
$$

where

$$
\begin{aligned}
& g_{0}\left(x_{k}\right)=\left[\frac{1}{\phi^{\prime}\left(x_{k}\right)}\right] \mu_{0}\left(x_{k}\right)-\left[\frac{\phi^{\prime \prime}\left(x_{k}\right)}{\left[\phi^{\prime}\left(x_{k}\right)\right]^{2}}\right] \mu_{1}\left(x_{k}\right)+\left[\frac{2\left[\phi^{\prime \prime}\left(x_{k}\right)\right]^{2}-\phi^{\prime \prime \prime}\left(x_{k}\right) \phi^{\prime}\left(x_{k}\right)}{\left[\phi^{\prime}\left(x_{k}\right)\right]^{3}}\right] \mu_{2}\left(x_{k}\right), \\
& g_{1}\left(x_{k}\right)=\mu_{1}\left(x_{k}\right)-\left[\frac{\phi^{\prime \prime}\left(x_{k}\right.}{\phi^{\prime}\left(x_{k}\right)}\right] \mu_{2}\left(x_{k}\right), \\
& g_{2}\left(x_{k}\right)=\phi^{\prime}\left(x_{k}\right) \mu_{2}\left(x_{k}\right) .
\end{aligned}
$$

Proof. We replace each term of (3.7) with its corresponding approximation given by (3.6) and (3.8) and substituting $x=x_{k}$,

$$
x_{k}=\phi^{-1}(k h)=\frac{e^{k h}}{1+e^{k h}}
$$

where the mesh size, $h$ is given by

$$
\mathrm{h}=\sqrt{\frac{\pi \mathrm{d}}{\alpha \mathrm{N}}}, \quad 0<\alpha \leqslant 1, \quad \mathrm{~d} \leqslant \frac{\pi}{2}
$$

where $N$ is suitably chosen and $\alpha$ depends on the asymptotic behavior of $u(x, t)$ and applying the collocation to it.

We use the boundary condition (3.4) and the quadrature formula of $F(x)$ given by

$$
\int_{a}^{b} F(x) d x=h \sum_{k=-N}^{N} \frac{F\left(x_{k}\right)}{\phi^{\prime}\left(x_{k}\right)}+O\left(e^{-(\pi \alpha d N)^{1 / 2}}\right) .
$$

We get two equations

$$
\begin{aligned}
& {\left[a_{0}\left(t_{i}\right)+\sum_{k=0}^{p} \alpha_{k}\left(t_{i}\right) \Phi_{1}\left(\epsilon_{k}\right)+h \sum_{j=-N}^{N} \frac{k_{1}\left(x_{j}, t_{i}\right)}{\phi^{\prime}\left(x_{j}\right)} \Phi_{1}\left(x_{j}\right)\right] c_{-N-2}^{i}} \\
& +\left[a_{1}\left(t_{i}\right)+\sum_{k=0}^{p} \alpha_{k}\left(t_{i}\right) \Phi_{3}\left(\epsilon_{j}\right)+h \sum_{j=-N}^{N} \frac{k_{1}\left(x_{j}, t_{i}\right)}{\phi^{\prime}\left(x_{j}\right)} \Phi_{3}\left(x_{j}\right)\right] c_{-N-1}^{i} \\
& +\sum_{j=-N}^{N}\left[h \frac{k_{1}\left(x_{j}, t_{i}\right)}{\left[\phi^{\prime}\left(x_{j}\right)\right]^{2}}+\sum_{k=0}^{p} \alpha_{k}\left(t_{i}\right) \frac{s_{j}\left(\epsilon_{k}\right)}{\phi^{\prime}\left(\epsilon_{k}\right)}\right] c_{j}^{i} \\
& +\left[a_{3}\left(t_{i}\right)+\sum_{k=0}^{p} \alpha_{k}\left(t_{i}\right) \Phi_{4}\left(\epsilon_{k}\right)+h \sum_{j=-N}^{N} \frac{k_{1}\left(x_{j}, t_{i}\right)}{\phi^{\prime}\left(x_{j}\right)} \Phi_{4}\left(x_{j}\right)\right] c_{N+1}^{i} \\
& +\left[a_{2}\left(t_{i}\right)+\sum_{k=0}^{p} \alpha_{k}\left(t_{i}\right) \Phi_{2}\left(\epsilon_{k}\right)+h \sum_{j=-N}^{N} \frac{k_{1}\left(x_{j}, t_{i}\right)}{\phi^{\prime}\left(x_{j}\right)} \Phi_{2}\left(x_{j}\right)\right] c_{N+2}^{i}=g_{1}\left(t_{i}\right),
\end{aligned}
$$


and

$$
\begin{aligned}
& {\left[b_{0}\left(t_{i}\right)+\sum_{k=0}^{p} \beta_{k}\left(t_{i}\right) \Phi_{1}\left(\xi_{k}\right)+h \sum_{j=-N}^{N} \frac{k_{2}\left(x_{j}, t_{i}\right)}{\phi^{\prime}\left(x_{j}\right)} \Phi_{1}\left(x_{j}\right)\right] c_{-N-2}^{i}} \\
& +\left[b_{1}\left(t_{i}\right)+\sum_{k=0}^{p} \beta_{k}\left(t_{i}\right) \Phi_{3}\left(\xi_{k}\right)+h \sum_{j=-N}^{N} \frac{k_{2}\left(x_{j}, t_{i}\right)}{\phi^{\prime}\left(x_{j}\right)} \Phi_{3}\left(x_{j}\right)\right] c_{-N-1}^{i} \\
& +\sum_{j=-N}^{N}\left[h \frac{k_{2}\left(x_{j}, t_{i}\right)}{\left[\phi^{\prime}\left(x_{j}\right)\right]^{2}}+\sum_{k=0}^{p} \beta_{k}\left(t_{i}\right) \frac{s_{j}\left(\xi_{k}\right)}{\phi^{\prime}\left(\xi_{k}\right)}\right] c_{j}^{i} \\
& +\left[b_{3}\left(t_{i}\right)+\sum_{k=0}^{p} \beta_{k}\left(t_{i}\right) \Phi_{4}\left(\xi_{k}\right)+h \sum_{j=-N}^{N} \frac{k_{2}\left(x_{j}, t_{i}\right)}{\phi^{\prime}\left(x_{j}\right)} \Phi_{4}\left(x_{j}\right)\right] c_{N+1}^{i} \\
& +\left[b_{2}\left(t_{i}\right)+\sum_{k=0}^{p} \beta_{k}\left(t_{i}\right) \Phi_{2}\left(\xi_{k}\right)+h \sum_{j=-N}^{N} \frac{k_{2}\left(x_{j}, t_{i}\right)}{\phi^{\prime}\left(x_{j}\right)} \Phi_{2}\left(x_{j}\right)\right] c_{N+2}^{i}=g_{2}\left(t_{i}\right) .
\end{aligned}
$$

Adding equations (3.10)-(3.11) to equation (3.9), then the discrete system for equations (3.2)-(3.4) is represented as

$$
\Lambda_{i} C_{i}=\Theta_{i}
$$

where

$$
\begin{aligned}
& \mathrm{C}_{i}=\left(\begin{array}{c}
c_{-N-2}^{i} \\
c_{-N-1}^{i} \\
c_{-N}^{i} \\
\vdots \\
\vdots \\
c_{N}^{i} \\
c_{N+1}^{i} \\
c_{N+2}^{i}
\end{array}\right) \quad \text { and } \quad \Theta_{i}=\left(\begin{array}{c}
g_{1}\left(t_{i}\right) \\
M\left(x_{-N-1}, t_{i}\right) \\
M\left(x_{-N}, t_{i}\right) \\
\vdots \\
\vdots \\
M\left(x_{N}, t_{i}\right) \\
M\left(x_{N+1}, t_{i}\right) \\
g_{2}\left(t_{i}\right)
\end{array}\right) \text {, }
\end{aligned}
$$

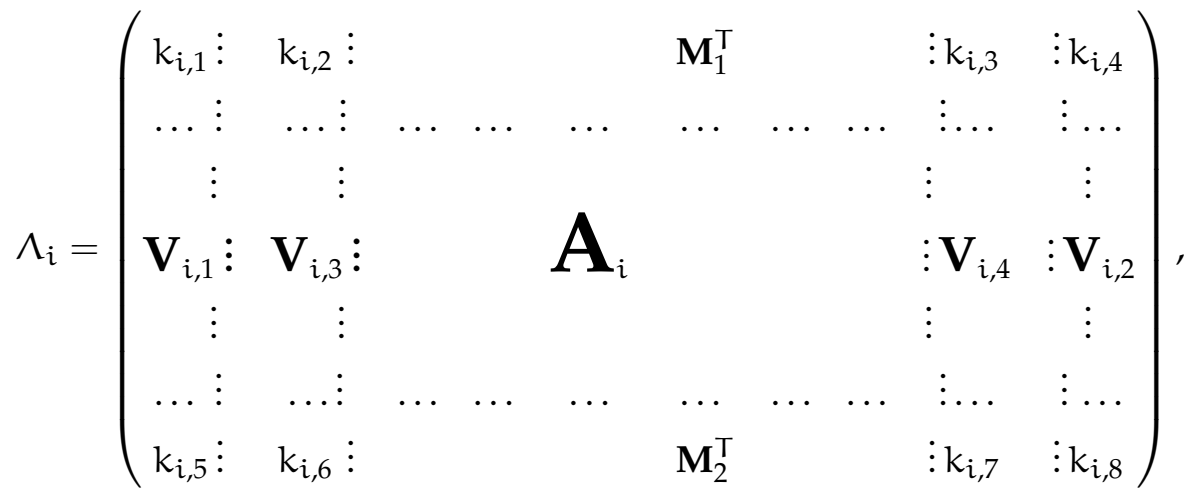

where

$$
k_{i, 1}=\left[a_{0}\left(t_{i}\right)+\sum_{k=0}^{p} \alpha_{k} \Phi_{1}\left(\varepsilon_{k}\right)+h \sum_{j=-N}^{N} \frac{k_{1}\left(x_{j}, t_{i}\right)}{\phi^{\prime}\left(x_{j}\right)} \Phi_{1}\left(x_{j}\right)\right],
$$




$$
\begin{aligned}
& k_{i, 2}=\left[a_{1}\left(t_{i}\right)+\sum_{k=0}^{p} \alpha_{k} \Phi_{3}\left(\varepsilon_{k}\right)+h \sum_{j=-N}^{N} \frac{k_{1}\left(x_{j}, t_{i}\right)}{\phi^{\prime}\left(x_{j}\right)} \Phi_{3}\left(x_{j}\right)\right], \\
& k_{i, 3}=\left[a_{3}\left(t_{i}\right)+\sum_{k=0}^{p} \alpha_{k} \Phi_{4}\left(\varepsilon_{k}\right)+h \sum_{j=-N}^{N} \frac{k_{1}\left(x_{j}, t_{i}\right)}{\phi^{\prime}\left(x_{j}\right)} \Phi_{4}\left(x_{j}\right)\right] \text {, } \\
& k_{i, 4}=\left[a_{2}\left(t_{i}\right)+\sum_{k=0}^{p} \alpha_{k} \Phi_{2}\left(\varepsilon_{k}\right)+h \sum_{j=-N}^{N} \frac{k_{1}\left(x_{j}, t_{i}\right)}{\phi^{\prime}\left(x_{j}\right)} \Phi_{2}\left(x_{j}\right)\right] \text {, } \\
& k_{i, 5}=\left[b_{0}\left(t_{i}\right)+\sum_{k=0}^{p} \beta_{k} \Phi_{1}\left(\xi_{k}\right)+h \sum_{j=-N}^{N} \frac{k_{2}\left(x_{j}, t_{i}\right)}{\phi^{\prime}\left(x_{j}\right)} \Phi_{1}\left(x_{j}\right)\right] \text {, } \\
& k_{i, 6}=\left[b_{1}\left(t_{i}\right)+\sum_{k=0}^{p} \beta_{k} \Phi_{3}\left(\xi_{k}\right)+h \sum_{j=-N}^{N} \frac{k_{2}\left(x_{j}, t_{i}\right)}{\phi^{\prime}\left(x_{j}\right)} \Phi_{3}\left(x_{j}\right)\right] \text {, } \\
& k_{i, 7}=\left[b_{3}\left(t_{i}\right)+\sum_{k=0}^{p} \beta_{k} \Phi_{4}\left(\xi_{k}\right)+h \sum_{j=-N}^{N} \frac{k_{2}\left(x_{j}, t_{i}\right)}{\phi^{\prime}\left(x_{j}\right)} \Phi_{4}\left(x_{j}\right)\right] \text {, } \\
& k_{i, 8}=\left[b_{2}\left(t_{i}\right)+\sum_{k=0}^{p} \beta_{k} \Phi_{2}\left(\xi_{k}\right)+h \sum_{j=-N}^{N} \frac{k_{2}\left(x_{j}, t_{i}\right)}{\phi^{\prime}\left(x_{j}\right)} \Phi_{2}\left(x_{j}\right)\right] \text {, }
\end{aligned}
$$

and $M_{i, 1}$ is $(2 N+1)$-vector with entries

$$
h \frac{k_{1}\left(t_{i}, x_{j}\right)}{\left[\phi^{\prime}\left(x_{j}\right)\right]^{2}}+\sum_{k=1}^{p} \alpha_{k} \frac{s_{j}\left(\varepsilon_{k}\right)}{\phi^{\prime}\left(\varepsilon_{k}\right)}, \quad j=-N, \ldots, N,
$$

and $M_{i, 2}$ is $(2 N+1)$-vector with entries

$$
h \frac{k_{2}\left(t_{i}, x_{j}\right)}{\left[\phi^{\prime}\left(x_{j}\right)\right]^{2}}+\sum_{k=1}^{p} \beta_{k} \frac{S_{j}\left(\xi_{k}\right)}{\phi^{\prime}\left(\xi_{k}\right)}, \quad j=-N, \ldots, N,
$$

also, $\mathbf{V}_{i, v}, v=1, \ldots, 4$ is a $(2 \mathrm{~N}+3)$-vector with entries $\left[\mathbf{L} \Phi_{v}\left(x_{k}\right)\right]$. The $(2 \mathbf{N}+3) \times(2 \mathrm{~N}+1)$ non-square matrix $A$ is given by

$$
\mathbf{A}_{i}=\sum_{p=0}^{2} \frac{1}{h^{p}} \mathbf{D}\left(g_{p}\right) \mathbf{I}^{(p)},
$$

where the matrices $\mathbf{I}^{(p)}, 0 \leqslant p \leqslant 2$ are given by

$$
\mathbf{I}^{(p)}=\left[\delta_{j k}^{(p)}\right],
$$

whose kj-th entry is given by [30]

$$
\delta_{j k}^{(0)}=\left\{\begin{array}{l}
1, j=k, \\
0, j \neq k,
\end{array} \quad \delta_{j k}^{(1)}=\left\{\begin{array}{l}
0, j=k, \\
\frac{(-1)^{k-j}}{k-j}, j \neq k,
\end{array} \quad \delta_{j k}^{(2)}=\left\{\begin{array}{l}
\frac{-\pi^{2}}{3}, j=k, \\
\frac{-2(-1)^{k-j}}{(k-j)^{2}}, j \neq k .
\end{array}\right.\right.\right.
$$

We have a linear system of $(2 N+5)$ equations for the $(2 N+5)$ unknown coefficients. By solving this linear system by the $\mathrm{Q}-\mathrm{R}$ method., the solution $\mathrm{C}_{i}$ gives the coefficients in the approximate sinc-collocation solution $u_{i, A}(x)$ of $u(x, t)$. 


\section{Numerical examples}

Four test examples from the open literature $[18,40,45]$ are constructed so as to clarify the accomplishment sinc-collocation method in finding a solution for parabolic PDE with nonlocal boundary conditions. For all examples, we take $d=\pi / 2$ and $\alpha=0.5$ and a sequence of runs for $N=5,10,20$ and 40 is reported. We measure the performance of sinc-collocation method by the maximum absolute error, $\left\|\mathrm{E}_{\text {sinc }}\right\|$, which is defined as

$$
\left\|E_{\text {sinc }}\right\|=\max \left|u_{\text {exact }}\left(x_{i}, t_{j}\right)-u_{A}\left(x_{i}, t_{j}\right)\right| .
$$

Example 4.1 ([18, 40]). Consider the equation

$$
u_{t}=u_{x x}-e^{-t}\left(x^{2}-x+\frac{\delta^{2}}{6\left(1+\delta^{2}\right)}+2\right)
$$

subject to the boundary conditions

$$
u(0, t)+\delta^{2} \int_{0}^{1} u(x, t) d x=0, \quad u(1, t)+\delta^{2} \int_{0}^{1} u(x, t) d x=0,
$$

and the initial condition

$$
u(x, 0)=x^{2}-x+\frac{\delta^{2}}{6\left(1+\delta^{2}\right)}
$$

whose the exact solution is

$$
u(x, t)=\exp (-t)\left(x^{2}-x+\frac{\delta^{2}}{6\left(1+\delta^{2}\right)}\right)
$$

where $\delta=0.12$. Table 1 presents maximum absolute error, $\left\|E_{\text {sinc }}\right\|$, at different times with $\Delta t=0.0001$ for different $\mathrm{N}$.

Table 1: $\left\|E_{\text {sinc }}\right\|$ for different $N$ for Example 4.1.

\begin{tabular}{ccccc}
\hline $\mathrm{N}$ & $\mathrm{T}=0.0001$ & $\mathrm{~T}=0.001$ & $\mathrm{~T}=0.01$ & $\mathrm{~T}=0.1$ \\
\hline 5 & $2.0720 \mathrm{E}-06$ & $2.3441 \mathrm{E}-06$ & $2.3520 \mathrm{E}-06$ & $1.3153 \mathrm{E}-05$ \\
10 & $2.0190 \mathrm{E}-08$ & $1.7473 \mathrm{E}-07$ & $1.8518 \mathrm{E}-06$ & $1.3710 \mathrm{E}-05$ \\
20 & $1.2370 \mathrm{E}-09$ & $1.7538 \mathrm{E}-07$ & $1.8598 \mathrm{E}-06$ & $1.3736 \mathrm{E}-05$ \\
40 & $1.9686 \mathrm{E}-08$ & $1.9430 \mathrm{E}-07$ & $1.8795 \mathrm{E}-06$ & $1.3752 \mathrm{E}-05$ \\
\hline
\end{tabular}

Example 4.2 ([45]). Consider the equation

$$
u_{t}=u_{x x}
$$

subject to the boundary conditions

$$
u(0, t)-\int_{0}^{1}(x+t) u(x, t) d x=g_{1}(t), \quad u(1, t)-\int_{0}^{1} t \exp (-x) u(x, t) d x=g_{2}(t),
$$

where

$$
\begin{aligned}
& g_{1}(t)=\frac{1}{2}-t-\exp (-t)[\cos 1+\sin 1+t \sin 1-2] \\
& g_{2}(t)=1+\exp (-t) \cos 1-\frac{t}{2 e}[2(e-1)+\exp (-t)(e-\cos 1+\sin 1)]
\end{aligned}
$$

and the initial condition

$$
u(x, 0)=1+\cos (x)
$$

whose the exact solution is

$$
u(x, t)=1+\exp (-t) \cos (x)
$$

Table 2 displays maximum absolute error, $\left\|E_{\text {sinc }}\right\|$, for different $N$ at different times with $\Delta t=0.0001$. 
Table 2: $\left\|\mathrm{E}_{\text {sinc }}\right\|$ for Example 4.2.

\begin{tabular}{ccccc}
\hline $\mathrm{N}$ & $\mathrm{T}=0.0001$ & $\mathrm{~T}=0.001$ & $\mathrm{~T}=0.01$ & $\mathrm{~T}=0.1$ \\
\hline 5 & $4.3205 \mathrm{E}-04$ & $4.6601 \mathrm{E}-04$ & $4.8697 \mathrm{E}-04$ & $6.7730 \mathrm{E}-04$ \\
10 & $4.2164 \mathrm{E}-05$ & $4.2431 \mathrm{E}-05$ & $4.3557 \mathrm{E}-05$ & $5.7325 \mathrm{E}-05$ \\
20 & $6.9375 \mathrm{E}-07$ & $8.2340 \mathrm{E}-07$ & $8.3762 \mathrm{E}-07$ & $3.0581 \mathrm{E}-06$ \\
40 & $4.9533 \mathrm{E}-09$ & $4.9354 \mathrm{E}-08$ & $4.7111 \mathrm{E}-07$ & $3.3727 \mathrm{E}-06$ \\
\hline
\end{tabular}

Example 4.3 ([45]). We consider the equation

$$
u_{t}=u_{x x}+\left(\pi^{2}-1\right) \exp (-t)[\sin (\pi x)+\cos (\pi x)], \quad 0<x<1, \quad 0<t<1,
$$

subject to the boundary conditions

$$
t^{2} u(0, t)+u_{x}(0, t)=\exp (-t)\left(t^{2}+\pi\right), \quad t u(1, t)+u_{x}(1, t)=-\exp (-t)(t+\pi),
$$

and the initial condition

$$
u(x, 0)=\sin (\pi x)+\cos (\pi x)
$$

whose the exact solution is

$$
u(x, t)=\exp (-t)[\sin (\pi x)+\cos (\pi x)] .
$$

Table 3 introduces maximum absolute error, $\left\|\mathrm{E}_{\text {sinc }}\right\|$, at different times.

Table 3: $\left\|\mathrm{E}_{\text {sinc }}\right\|$ for Example 4.3.

\begin{tabular}{ccccc}
\hline $\mathrm{N}$ & $\mathrm{T}=0.0001$ & $\mathrm{~T}=0.001$ & $\mathrm{~T}=0.01$ & $\mathrm{~T}=0.1$ \\
\hline 5 & $7.3909 \mathrm{E}-05$ & $3.2724 \mathrm{E}-04$ & $1.1537 \mathrm{E}-03$ & $3.4481 \mathrm{E}-03$ \\
10 & $7.3355 \mathrm{E}-06$ & $3.5886 \mathrm{E}-05$ & $1.2458 \mathrm{E}-04$ & $2.2470 \mathrm{E}-04$ \\
20 & $2.7744 \mathrm{E}-07$ & $1.1029 \mathrm{E}-06$ & $3.0855 \mathrm{E}-06$ & $7.8614 \mathrm{E}-06$ \\
40 & $8.1663 \mathrm{E}-09$ & $7.8325 \mathrm{E}-08$ & $6.8562 \mathrm{E}-07$ & $5.5275 \mathrm{E}-06$ \\
\hline
\end{tabular}

Example 4.4 ([40]). We consider the equation

$$
u_{t}=u_{x x}+[2 \exp (-t)-t \exp (-t)-1][1+x], \quad 0<x<1, \quad 0<t<1,
$$

subject to the boundary conditions

$$
u(0, t)-\frac{6}{5} \int_{0}^{1} x u(x, t) d x=0, \quad u(1, t)-\frac{4}{3} \int_{0}^{1} u(x, t) d x=0,
$$

and the initial condition

$$
u(x, 0)=0,
$$

whose the exact solution is

$$
\mathrm{u}(\mathrm{x}, \mathrm{t})=[1-\exp (-\mathrm{t})-\mathrm{t}+\mathrm{t} \exp (-\mathrm{t})](1+\mathrm{x}) .
$$

Table 4 displays maximum absolute error, $\left\|\mathrm{E}_{\text {sinc }}\right\|$, at different times with $\Delta t=0.0001$.

Table 4: $\left\|\mathrm{E}_{\text {sinc }}\right\|$ at different times for Example 4.4.

\begin{tabular}{ccccc}
\hline $\mathrm{N}$ & $\mathrm{T}=0.0001$ & $\mathrm{~T}=0.001$ & $\mathrm{~T}=0.01$ & $\mathrm{~T}=0.1$ \\
\hline 5 & $1.7365 \mathrm{E}-07$ & $1.4629 \mathrm{E}-06$ & $1.5220 \mathrm{E}-05$ & $2.2710 \mathrm{E}-04$ \\
10 & $4.1473 \mathrm{E}-08$ & $2.2598 \mathrm{E}-07$ & $2.5054 \mathrm{E}-06$ & $1.8095 \mathrm{E}-05$ \\
20 & $3.0220 \mathrm{E}-08$ & $2.3751 \mathrm{E}-07$ & $2.8946 \mathrm{E}-06$ & $2.7668 \mathrm{E}-05$ \\
40 & $2.9998 \mathrm{E}-08$ & $2.3980 \mathrm{E}-07$ & $2.9200 \mathrm{E}-06$ & $2.8021 \mathrm{E}-05$ \\
\hline
\end{tabular}




\section{Conclusions}

In this paper, we presented a simple-to-implement yet accurate method using sinc-collocation scheme. In view of the aforementioned numerical results, the accuracy of this method is considerably manifest. As a consequence, the suggested method is effective in solving this problem. We believe that the current method can be further developed to treat the more realistic physical models where the system has more than one space variable.

\section{References}

[1] A. Abdrabou, M. El-Gamel, On the sinc-Galerkin method for triharmonic boundary-value problems, Comput. Math. Appl., 76 (2018), 520-533. 2

[2] W. Allegretto, Y. P. Lin, A. Zhou, A box scheme for coupled systems resulting from microsensor thermistor problems, Dynam. Contin. Discrete Impuls. Systems, 5 (1999), 209-223. 1

[3] M. Bastani, D. k. Khojasteh, Numerical studies of a non-local parabolic partial differential equations by spectral collocation method with preconditioning, Comput. Math. Modeling, 24 (2013), 81-89. 1

[4] B. Bialecki, Sinc-collocation methods for two-point boundary value problems, IMA J. Numer. Anal., 11 (1991), $357-375$. 1

[5] A. Bouziani, Mixed problem with boundary integral conditions for a certain parabolic equation, J. Appl. Math. Stochastic Anal., 9 (1996), 323-330. 1

[6] A. Bouziani, Strong solution for a mixed problem with nonlocal condition for a certain pluriparabolic equations, Hiroshima Math. J., 27 (1997), 373-390.

[7] A. Bouziani, On a class of parabolic equations with a nonlocal boundary condition, Acad. Roy. Belg. Bull. Cl. Sci. (6), 10 (1999), 61-77. 1

[8] A. Bouziani, N. Merazga, S. Benamira, Galerkin method applied to a parabolic evolution problem with nonlocal boundary conditions, Nonlinear Anal., 69 (2008), 1515-1524. 1

[9] J. R. Cannon, The solution of the heat equation subject to the specification of energy, Quart. Appl. Math., 21 (1963), 155-160. 1

[10] J. R. Cannon, Y. P. Lin, S. M. Wang, An implicit finite difference scheme for the diffusion equation subject to mass specification, Int. J. Engrg. Sci., 28 (1990), 573-578. 1

[11] J. R. Cannon, A. L. Matheson, A numerical procedure for diffusion subject to the specification of mass, Int. J. Engrg. Sci., 31 (1993), 347-355.

[12] J. R. Cannon, S. Perez Esteva, J. van der Hoek, A Galerkin procedure for the diffusion equation subject to the specification of mass, SIAM J. Numer. Anal., 24 (1987), 499-515. 1

[13] J. H. Cushman, T. R. Ginn, Nonlocal dispersion in porous media with continuously evolving scales of heterogeneity, Transp. Porous Media, 13 (1993), 123-138. 1

[14] J. H. Cushman, H. X. Xu, F. W. Deng, Nonlocal reactive transport with physical and chemical heterogeneity: localization error, Water Resources Res., 31 (1995), 2219-2237. 1

[15] G. Dagan, The significance of heterogeneity of evolving scales to transport in porous formations, Water Resources Res., 13 (1994), 3327-3336. 1

[16] W. A. Day, A decreasing property of solutions of parabolic equations with applications to thermoelasticity, Quart. Appl. Math., 40 (1982), 468-475. 1

[17] W. A. Day, Extensions of a property of the heat equation to linear thermoelasticity and other theories, Quart. Appl. Math., 41 (1982/83), 319-330. 1

[18] G. Ekolin, Finite difference methods for a nonlocal boundary value problem for the heat equation, BIT, 31 (1991), $245-261$. $1,4,4.1$

[19] M. El-Gamel, A numerical scheme for solving nonhomogeneous time-dependent problems, Z. Angew. Math. Phys., 57 (2006), 369-383. 1

[20] M. El-Gamel, Numerical solution of Troesch's problem by sinc-collocation method, Appl. Math., 4 (2013), 707-712. 1

[21] M. El-Gamel, A note on solving the fourth-order parabolic equation by the sinc-Galerkin method, Calcolo, 52 (2015), 327-342. 2

[22] M. El-Gamel, Error analysis of sinc-Galerkin method for time-dependent partial differential equations, Numer. Algorithms, 77 (2018), 517-533. 2

[23] M. El-Gamel, A. Abdrabou, Sinc-Galerkin solution to eighth-order boundary value problems, SeMA J., 76 (2019), 249 270. 1

[24] M. El-Gamel, A. I. Zayed, A comparison between the wavelet-Galerkin and the Sinc-Galerkin methods in solving nonhomogeneous heat equations, in: Inverse Problem, Image Analysis, and Medical Imaging, 2002 (2002), 97-116. 1

[25] R. Ewing, R. Lazarov, Y. P. Lin, Finite volume element approximations of nonlocal reactive flows in porous media, Numer. Methods Partial Differential Equations, 16 (2000), 285-311. 1

[26] G. Fairweather, R. D. Saylor, The reformulation and numerical solution of certain nonclassical initial-boundary value problems, SIAM J. Sci. Statist. Comput., 12 (1991), 127-144. 1 
[27] A. Golbabai, M. Javidi, A numerical solution for nonclassical parabolic problem based on Chebyshev spectral collocation method, Appl. Math. Comput., 190 (2007), 179-185. 1

[28] V.-M. Hokkanen, G. Morosanu, Functional Methods in Differential Equations, Chapman \& Hall/CRC, Boca Raton, (2002). 1

[29] N. Ionkin, Solution of a boundary value problem in heat conduction with a non-classical boundary condition, Differential Equations, 13 (1977), 204-211. 1

[30] J. Lund, K. L. Bowers, Sinc Methods for Quadrature and Differential Equations, SIAM, Philadelphia, (1992). 2, 3

[31] A. M. Nakhushev, On certain approximate method for boundary-value problems for differential equations and its applications in ground waters dynamics, Differ. Uravn., 18 (1982), 72-81. 1

[32] M. K. Ng, Fast iterative methods for symmetric sinc-Galerkin system, IMA J. Numer. Anal., 19 (1999), 357-373. 1

[33] A. K. Pani, A finite element method for a diffusion equation with constrained energy and nonlinear boundary conditions, J. Austral. Math. Soc. Ser. B, 35 (1993), 87-102. 1

[34] M. Renardy, W. J. Hrusa, J. A. Nohel, Mathematical Problems in Viscoelasticity, John Wiley \& Sons, New York, (1987). 1

[35] A. Samarskii, Some problems in differential equations theory, Differential Equations, 16 (1980), 1221-1228. 1

[36] R. C. Smith, G. A. Bogar, K. L. Bowers, J. Lund, The Sinc-Galerkin method for fourth-order differential equations, SIAM J. Numer. Anal., 28 (1991), 760-788. 1

[37] F. Stenger, A "sinc-Galerkin" method of solution of boundary value problems, Math. Comput., 33 (1979), 85-109. 1

[38] F. Stenger, Numerical Methods Based on Sinc and Analytic Functions, Springer-Verlag, New York, (1993). 2

[39] F. Stenger, Summary of sinc numerical methods, J. Comput. Appl. Math., 121 (2000), 379-420. 1

[40] M. Tadi, M. Radenkovic, A numerical method for 1-D parabolic equation with nonlocal boundary conditions, Int. J. Comput. Math., 2014 (2014), 9 pages. 4, 4.1, 4.4

[41] A. S. Vasudeva Murthy, J. G. Verwer, Solving parabolic integro-differential equations by an explicit integration method, J. Comput. Appl. Math., 39 (1992), 121-132.

[42] V. Vodakhova, A boundary-value problem with nonlocal condition for certain pseudo-parabolic water-transfer equation, Differentsialnie Uravnenia, 18 (1982), 280-285. 1

[43] G. Y. Yin, Sinc-Collocation method with orthogonalization for singular problem-like poisson, Math. Comp., 62 (1994), 21-40. 1

[44] S. A. Yousefi, M. Behroozifar, M. Dehghan, The operational matrices of Bernstein polynomials for solving the parabolic equation subject to specification of the mass, J. Comput. Appl. Math., 235 (2011), 5272-5283. 1

[45] R. Zolfaghari, A. Shidfar, Solving a parabolic PDE with nonlocal boundary conditions using the Sinc method, Numer. Algorithms, 62 (2013), 411-427. 1, 4, 4.2, 4.3 